\title{
Association of Infant Feeding Practices with Iron Status and Hematologic Parametersin Thai Infants at 6 Months of Age
}

\section{Chayatat Ruangkit}

Ramathibodi Medical School, Chakri Naruebodindra Medical Institute, Faculty of Medicine Ramathibodi Hospital, Mahidol University, Samut Prakan

\section{Nawapat Prachakittikul}

Chakri Naruebodindra Medical Institute, Faculty of Medicine Ramathibodi Hospital, Mahidol University,

Samut Prakan

\section{Nutthida Hemprachitchai}

Chakri Naruebodindra Medical Institute, Faculty of Medicine Ramathibodi Hospital, Mahidol University, Samut Prakan

\section{Oraporn Dumrongwongsiri}

Department of Pediatrics, Faculty of Medicine Ramathibodi Hospital, Mahidol University, Bangkok

Sasivimon Soonsawad ( $\sim$ sasivimon.soo@mahidol.ac.th )

Ramathibodi Medical School, Chakri Naruebodindra Medical Institute, Faculty of Medicine Ramathibodi Hospital, Mahidol University, Samut Prakan

\section{Research Article}

Keywords: Infant feeding, breastfeeding, formula feeding, iron supplements, iron deficiency anemia

Posted Date: September 28th, 2021

DOl: https://doi.org/10.21203/rs.3.rs-934107/v1

License: (c) (i) This work is licensed under a Creative Commons Attribution 4.0 International License. Read Full License 


\section{Association of infant feeding practices with iron status and hematologic}

\section{2 parameters in Thai infants at 6 months of age}

3 Chayatat Ruangkit, M.D. ${ }^{a}$, Nawapat Prachakittikul, M.D. ${ }^{b}$, Nutthida Hemprachitchai, M.D. ${ }^{b}$,

4 Oraporn Dumrongwongsiri, M.D. ${ }^{\mathrm{c}}$, Sasivimon Soonsawad, M.D. ${ }^{\mathrm{a}}$

\section{Affiliations}

6 a. Ramathibodi Medical School, Chakri Naruebodindra Medical Institute, Faculty of

7 Medicine Ramathibodi Hospital, Mahidol University, Samut Prakan, Thailand

8 b. Chakri Naruebodindra Medical Institute, Faculty of Medicine Ramathibodi Hospital,

9 Mahidol University, Samut Prakan, Thailand

c. Department of Pediatrics, Faculty of Medicine Ramathibodi Hospital, Mahidol University,

11 Bangkok, Thailand

12 Corresponding author: Sasivimon Soonsawad, M.D., 111 Suvarnabhumi Canal Road, Bang

13 Pla, Bang Phli District, Samut Prakan 10540, Thailand

14 E-mail: sasivimon.soo@mahidol.ac.th

15 Phone: $(+66)$ 097-012-3560

\section{Running Title}




\section{Abstract}

Background: An infant's iron intake in the first 6 months of life comes solely from milk

intake. However, infants' feeding practices vary, and their association with infants' iron status and hematologic parameters has not been well studied. We aimed to evaluate how different infant feeding practices associate with iron status and hematologic parameters among 6month-old Thai infants.

Methods: In a retrospective chart review, we identified 403 infants who attended a well-baby clinic and received laboratory screening for anemia (complete blood count and serum ferritin) at 6-month visits. Infants were categorized into four groups according to feeding practices. Hematologic parameters and incidence of anemia (hemoglobin $[\mathrm{Hb}]<11 \mathrm{~g} / \mathrm{dL}$ ), iron deficiency (ID; ferritin $<12 \mathrm{ng} / \mathrm{mL}$ ), and iron deficiency anemia (IDA; $\mathrm{Hb}<11 \mathrm{~g} / \mathrm{dL}$ and ferritin $<12 \mathrm{ng} / \mathrm{mL}$ ) were compared between groups. Univariate and multiple logistic regression models were used to identify IDA associated factors among 6-month-old infants.

Results: In total, 105 infants were breastfed (BF), 78 were breastfed with iron supplementation (BI), 109 infants were mixed-fed (breast milk and formula) with or without iron supplementation starting at age 4 months (MF), and 111 infants were formula-fed (FF). The BF group had the highest incidence of anemia, ID, and IDA. Anemia was found in $38.1 \%$ of BF infants compared with $21.8 \%$ of BI, $19.3 \%$ of MF, and $16.2 \%$ of FF infants $(p<0.001)$. ID was found in $28.6 \%$ of BF infants compared with $3.8 \%$ of BI, $3.7 \%$ of MF, and $0.9 \%$ of FF infants $(\mathrm{p}<0.001)$. IDA was found in $17.1 \%$ of BF infants compared with $2.6 \%$ of BI, $0.9 \%$ of $\mathrm{MF}$, and $0.9 \%$ of $\mathrm{FF}$ infants $(\mathrm{p}<0.001)$. In multivariate logistic regression, higher weight gain during 0-6 months slightly increased the risk of IDA and higher birth weight slightly decreased this risk. BI, MF, and FF infants had 90.4\%, 97.5\%, and 96.9\% decreased risk of IDA, respectively, with BF infants as a reference group. 
46 Conclusion: The incidence of anemia, ID, and IDA at age 6 months was higher in BF than

47 FF or partially BF infants. However, iron supplements in BF infants starting at 4 months

48 significantly reduced their ID and IDA incidence.

\section{Keywords}

50 Infant feeding, breastfeeding, formula feeding, iron supplements, iron deficiency anemia

51

52

53

54

55

56

57

58

59

60

61

62

63

64

65 
Iron deficiency (ID), one of the most common nutritional deficiencies, remains a significant global public health challenge in developed and developing countries [1]. Because iron is an essential component of heme protein, the main component of red blood cells, ID is the most common cause of anemia worldwide [2, 3]. Iron deficiency anemia (IDA) deleteriously affects many body systems and organ functions. Children with IDA can be asymptomatic or display non-specific symptoms such as loss of appetite, being easily fatigued, or growth retardation. In severe IDA, children can present with lethargy, hepatosplenomegaly, frequent infection or heart failure [4]. Among the most concerning detrimental effects of IDA in children are those involving behavior, cognition, and psychomotor skills. Several previous studies have indicated an association between ID in infants and poor neurodevelopmental outcomes. For example, a 1987 study of 191 Costa Rican children by Lozoff et al. found that children with IDA had lower developmental test scores than children without this condition [5]. In their follow-up study, many children who had severe, chronic ID in infancy continued to experience behavioral and developmental problems 10 years later, although their anemia had been corrected [6].

A full-term infant has iron stores at birth that are sufficient until 4-6 months of age.

During the first 6 months of life, infant iron status depends more on the iron store at birth than the iron intake from breast milk. The iron content in breast milk is low and decreases over the lactation period. Nevertheless, because breast milk is considered the best nutrition for infants during the first year of life, especially in the first 6 months, the World Health Organization (WHO) and United Nations Children's Fund (UNICEF) promote breastfeeding as a global public health policy [7]. In Thailand, The Royal College of Pediatricians of 
infants under 6 months of age be exclusively breastfed, with no need for complementary feeding or vitamin and mineral supplementation $[8,9]$. Previous studies on iron status in infants have shown that anemia and IDA occur more frequently in breastfed infants than those who are formula-fed $[10,11]$. A cross-sectional study of iron status in breastfed infants age 3-5 months showed a higher prevalence of ID and IDA among 5-month-old compared with 3-month-old infants [12]. A longitudinal study following the iron status of healthy breastfed infants at 4 and 6 months of age showed an increase in the prevalence of ID (5.7\% to $26.1 \%$ ) and IDA (3.4\% to $23.9 \%$ ) among infants at 4 months compared with 6 months [13]. These findings raise questions regarding iron adequacy among breastfed infants during the first 4-6 months of life. Consequently, although fully supporting breastfeeding, the American Academy of Pediatrics (AAP) recommended in 2010 that exclusively breastfed infants be given $1 \mathrm{mg} / \mathrm{kg}$ /day iron supplementation after 4 months of age to prevent IDA [14]. However, the risks and benefits of this practice remain inconclusive.

To our knowledge, there is limited information regarding iron status among Thai infants during the first 6 months of life, which is the age before iron-rich complementary feeding or iron supplementation should be introduced, as recommended by many health organizations. However, because The Royal College of Pediatricians of Thailand and Pediatric Society of Thailand had suggested universal screening for anemia among infants between age 6 and 12 months [15], many studies have investigated the iron status of infants during this period. The findings show that $34.0 \%-63.5 \%$ of infants have serum ferritin $<30$ $\mathrm{ng} / \mathrm{mL}$, which is defined as within the range of insufficiency to deficiency [16, 17]. Data in this patient age group also suggest that IDA is associated with low birth weight, low dietary iron intake, low household income, and long duration of breastfeeding [17-19]. It is possible that the practice of anemia screening during late infancy (9-12 months of age), which is 
commonly performed in many institutions, may be too late; anemia may occur earlier because the neonatal iron store is exhausted before 6 months.

Because feeding is the primary source of iron intake for infants, feeding practices considerably impact their iron status. Infant feeding practices during the first 6 months of life vary widely. Some infants are exclusively breastfed whereas others are given infant formula or a combination of both breast milk and formula. Although iron supplementation for breastfed infants, as recommended in the AAP guideline, is not endorsed by Thai health supplementation may have a preventive effect on ID in exclusively or partially breastfed infants. Some infants may also be given complementary foods early, before they have reached 6 months of age.

In this study, we investigated the association of infant feeding practices with iron status and hematologic parameters among infants at 6 months of age using historical data of patients examined in a well-baby clinic. Our primary objective was to compare the prevalence of anemia, ID, and IDA at 6 months of age between infants who received different types of feeding (breast milk only, breast milk with iron supplements, a combination of breast milk and infant formula with or without iron supplements, and infant formula only). The secondary objective was to identify possible factors associated with IDA at 6 months of age in these infants.

\section{Methods}

We performed a retrospective cohort study and reviewed the electronic medical records of healthy infants visiting the well-baby clinic at Chakri Naruebodindra Medical Institute (CNMI), Samut Prakan, Thailand, from January 1, 2019 to December 30, 2020. This 
study was approved by the Faculty of Medicine Ramathibodi Hospital, Mahidol University Ethics Committee (register No. MURA2021/44). The study was performed in accordance with the International Ethical Guidelines for Biomedical Research Involving Human Subjects and ethical principles of the Declaration of Helsinki. A waiver of individual patient informed consent was granted.

During the study period, a protocol for anemia screening in 6-month-old infants was implemented in the well-baby clinic at CNMI, including complete blood count (CBC) and serum ferritin measurement. This screening protocol was in compliance with guidelines of the Royal College of Pediatrics of Thailand and the Pediatrics Society of Thailand, which recommend checking hemoglobin $(\mathrm{Hb})$ or hematocrit $(\mathrm{HCT})$ at least once in infants from 6 to 12 months of age [15]. All patients with results of serum ferritin measurement performed during the study period were identified in the CNMI laboratory electronic database. Only infants age 5-7 months with $\mathrm{CBC}$ and serum ferritin results were selected for chart review. Only the medical records of infants with available information from 4-month and 6-month well-baby visits in the electronic database were included in the study. Premature infants with gestational age at birth less than 35 weeks and infants who received iron supplements before 4 months of age were excluded from the study.

A self-administered questionnaire is routinely provided to the parents of infants visiting a well-baby clinic at CNMI, to be completed before each physician encounter. These questionnaires, which are tailored according to infants' age (2 months, 4 months, or 6 months), specifically query the parents about current infant feeding practices and developmental milestones, as well as parents' general knowledge about child-rearing. Infant weight, length, and head circumference measurements are performed by skilled nurses at the clinic before each physician encounter. During each visit with a pediatrician, the infant's anthropometric data, together with a patient history and findings of physical examination 
performed by the pediatrician, are recorded in the form of a physician's note. At the end of each visit, the questionnaire and physician's note are entered into the electronic medical records database. Data extraction was performed using these documents. Data collection consisted of infants' baseline characteristics, including sex, gestational age, weight, length, and head circumference at birth, and the data from continuous infant monitoring at the wellbaby clinic at 4 and 6 months of age including weight, length, head circumference, and infant feeding practice. We collected laboratory results, including $\mathrm{CBC}$ and serum ferritin levels at 6 months of age. Infants were categorized into four groups according to feeding practices: 1) breastfed infants (BF) were those whose parents reported in the 4-month and 6-month questionnaires that the infant was fed breast milk (without infant formula or iron supplements);2) breastfed with iron supplements (BI) referred to infants whose parents documented in the 4-month and 6-month questionnaires that the infant was fed breast milk and also received iron supplements prescribed by a physician starting at the 4-month visit; 3) mixed-fed with or without iron supplements (MF) comprised infants whose parents reported in either the 4- or 6-month questionnaire that the infant was fed breast milk and infant formula together or was fed breast milk at the 4-month visit and fed infant formula at the 6month visit (infants in this group may or may not have received iron supplementation); and 4) formula-fed infants (FF) were those whose parents documented in the 4-month and 6-month questionnaires that their infant was fed infant formula (without breast milk or iron supplementation). There were two pediatric iron supplement products available in the hospital at the time of the study; ferrous fumarate suspension with $15 \mathrm{mg}$ of elemental iron per $0.6 \mathrm{~mL}$, and iron (III) hydroxide polymaltose complex syrup with $10 \mathrm{mg}$ of elemental iron per $1 \mathrm{~mL}$. All infants who received iron supplementation were prescribed either of these products at the dose of $1-2 \mathrm{mg} / \mathrm{kg} /$ day of elemental iron. 


\section{Biochemical analyses}

189

190

191

192

193

194

195

196

197

198

199

200

201

202

203

204

205

206

207

208

209

210

211

212

CBCs were conducted using an automated hematology analyzer (Sysmex XN 3000; Sysmex Asia Pacific Pte Ltd, Jalan Kilang, Singapore). Serum ferritin concentrations were measured using a sandwich-type electrochemiluminescence immune assay (Cobas 6000; Roche Diagnostics, Basel, Switzerland), with a measurement range of $0.5-2000 \mathrm{ng} / \mathrm{mL}$. Internal quality control runs were performed daily, per manufacturer guidelines. All external quality assessment was performed according to the Bio-Rad Laboratories Ltd. EQAS program, accredited to ISO 17043:2010. In this study, iron deficiency (ID) in infants was defined as serum ferritin $<12 \mathrm{ng} / \mathrm{mL}$, anemia in infants was defined as $\mathrm{Hb}<11 \mathrm{~g} / \mathrm{dL}$, and iron deficiency anemia (IDA) in infants was defined as both serum ferritin $<12 \mathrm{ng} / \mathrm{mL}$ and $\mathrm{Hb}<11 \mathrm{~g} / \mathrm{dL}$ [20]. Biochemical markers for iron status can be altered by recent infection and inflammation; however, inflammatory markers were not determined in the well-child setting under the assumption that infants were healthy when they presented for health check-ups and vaccination.

\section{Statistical analysis}

We performed univariate analyses to identify significant differences between the groups. One-way analysis of variance and post-hoc Bonferroni tests were used for parametric continuous variables, and results are presented as mean \pm standard deviation. Kruskal-Wallis and post-hoc Mann-Whitney U tests were used for non-parametric continuous variables. Results are presented as median (interquartile range). Pearson's chi-square or Fisher exact tests were used for categorical variables (pre-hoc and post-hoc pairwise analysis), and the results are presented as number (\%). To determine the factors associated with IDA in infants at 6 months old, univariate logistic regression was performed to investigate the simple association between each variable with infant IDA. Factors demonstrating an association with 
$\mathrm{p}<0.1$ were entered into the multivariate logistic regression model. Feeding practices was included in the univariate and multivariate logistic regression analysis, and the BF group was set as the reference group. A p-value $<0.05$ was considered statistically significant. However, the Bonferroni-adjusted p-value for significance of 0.008 was set for post-hoc (pairwise) analysis of categorical variables. Statistical analyses were performed using SPSS version 18.0 (SPSS Inc, Chicago, IL, USA).

\section{Results}

During the study period, 403 infants met the inclusion criteria and were enrolled in this study. The infants were categorized according to four feeding patterns, as follows: 105 infants in the BF group, 78 infants in the BI group, 109 infants in the MF group, and 111 infants in the FF group. Figure 1 shows the number of study participants and the reasons for exclusion.

There were no significant differences in infants' baseline characteristics between the four groups. Complementary feeding was introduced before 6 months of age in $31.1 \%$ of BF, $53.1 \%$ of BI, $79.8 \%$ of MF, and $85.7 \%$ of FF infants. All infants $(100 \%)$ in the BI group and $40(36.7 \%)$ in the MF group received iron supplements, started at the 4-month visit, whereas no infants in the BF and FF groups received iron supplements (Table 1).

The mean age of infants at the time of laboratory evaluation was $189 \pm 11$ days in BF, $190 \pm 11$ days in BI, 192 \pm 11 days in MF, and $193 \pm 9$ days in FF infants, respectively. Laboratory findings at 6 months showed that infants in the BF group had significantly lower mean serum ferritin levels, $\mathrm{Hb}, \mathrm{HCT}$, mean corpuscular volume (MCV), mean corpuscular hemoglobin $(\mathrm{MCH})$, and mean corpuscular hemoglobin concentration (MCHC) in comparison with the other groups. In contrast, the mean red blood cell distribution width (RDW) of infants in the BF group was significantly higher than that in the other groups. 
$17.1 \%$ had IDA, which were all significantly higher than these proportions in the other

groups. In contrast, there was no significant difference in the rate of infants who had anemia

without ID between the four groups (Table 2).

Univariate logistic regression analysis was performed to determine the predictive

factors for IDA in 6-month-old infants. Male sex and greater weight gain during 0-6 months

were associated with increased risk of IDA whereas older gestational age and higher birth associated with decreased risk of IDA, with BF infants as the reference group. In contrast, small for gestational age and complementary feeding initiated before 6 months were not associated with the risk of IDA. In the multivariate logistic regression model, which included factors with $\mathrm{p}<0.1$ in univariate logistic regression analysis, greater weight gain during 0-6 months slightly increased the risk of IDA and higher birth weight slightly decreased this risk. Infant feeding patterns strongly predicted IDA in the multivariate logistic regression model. With BF infants as the reference group, the BI, MF, and FF groups had 90.4\%, 97.5\%, and 96.9\% decreased risk of IDA, respectively (Table 3 ).

\section{Discussion}

Our study found that $28.6 \%$ and $17.1 \%$ of Thai infants who were breastfed but did not receive iron supplementation had ID and IDA, respectively, at 6 months of age. These incidences were significantly higher than those among formula-fed or partially breastfed infants. We found that breastfed infants who received $1-2 \mathrm{mg} / \mathrm{kg} /$ day of iron supplementation starting at 4 months of age had iron status and hematologic parameters comparable to those of formula-fed or partially breastfed infants. Infants in the BF group had significantly lower mean serum ferritin levels and significant differences in hematologic parameters compatible 
with IDA (low Hb, HCT, MCV, MCH, MCHC, and high RDW) in comparison with infants with other feeding patterns.

When infants in all groups were combined, the overall prevalence of anemia in 6month-old infants was $23.9 \%$. which is comparable to the prevalence previously reported in multiple studies among infants and children in similar age groups in Thailand. A study by Suwannakeeree et al. found a $29.1 \%$ prevalence of anemia in 9-month-old infants [19], and Tantracheewathorn et al. found 26.4\% prevalence in 9-12 month-old infants [18].

Rojroongwasinkul et al. reported a $26.0 \%$ prevalence in urban children age between 0.5 to 2.9 years in the South East Asian Nutrition Survey [21]. The overall prevalence of IDA among infants in our study was $5.5 \%$, which was lower than previously reported prevalence among 9-12 month-old infants in Thailand $(14.3 \%$ by Tantracheewathorn et al. and $17.9 \%$ by Suwannakeeree et al.) $[18,19]$. This difference in IDA prevalence was likely owing to differences in the ages of the study populations. The iron store at birth is the source for iron utilization in infants until approximately 6 months of age. The risk of IDA among infants over 6 months old is increased unless appropriate complementary feeding is given. Additionally, the feeding patterns differed between studies. Our study included a large number of formula-fed infants, many of who were breastfed or partially breastfed and receiving iron supplements beginning at 4 months old, which may help to prevent IDA. In our study, $15 \%-20 \%$ of infants in each group were anemic despite having normal serum ferritin levels at 6 months. Anemia among these infants was unlikely to have been caused by ID. Most anemic infants in our study with serum ferritin levels above the cut-point may have thalassemia and hemoglobinopathy genes because thalassemia is endemic in Thailand. A study among healthy infants age 6-12 months in the northern part of Thailand showed that $29.4 \%$ of infants were thalassemia carriers and $2.4 \%$ had thalassemia disease [22]. Other nutritional deficiencies such as vitamin A, vitamin B12, and folate, or parasitic 
diseases or infestation such as malaria or hookworm, could also contribute to anemia in infants [23]. Unfortunately, owing to the lack of consistency in patient management, followup, and further laboratory investigation, the cause of anemia in these infants could not be confirmed.

Previous studies have revealed evidence linking exclusive breastfeeding during the first 6 months of life or longer to an increased risk of IDA among infants at 6-12 months of age $[17-19,24]$. Current practice guidelines from the Ministry of Health of Thailand recommend weekly iron supplements (12.5 $\mathrm{mg}$ of elemental iron once a week) for infants starting at 6 months of age [25]. This recommendation can ensure the iron status of infants older than 6 months of age, but the adequacy of iron for infants during the first 4-6 months of life remains unclear. As demonstrated in our study, approximately one in every four breastfed infants had ID at 6 months of age, and nearly one in every five breastfed infants had IDA. Given the potential negative effects of even pre-anemic ID on early development, waiting until infants reach 6 months of age before starting supplementation may be too late. A metaanalysis of iron supplementation among breastfed infants at an early age showed limited evidence regarding the effect of iron supplements in the prevention of IDA [26]. However, the impact of supplements depends on the initial infant iron status, as shown in a randomized control trial that compared the prevalence of IDA among exclusively breastfed infants with iron supplementation during ages 4-9 and 6-9 months. That study was conducted in two areas (Honduras and Sweden) with relatively different amounts of dietary iron consumption. Iron supplementation in breastfed infants from 4 months effectively reduced the prevalence of ID at 6 months of age only in Honduras, where the baseline prevalence of ID is high and dietary iron intake is low [27]. Neonatal iron storage has a more significant effect on serum ferritin levels in early infancy than iron consumption from breast milk [28]. Our study findings imply that a considerable number of breastfed infants who attend our clinic may have low iron 
stores at birth, which are affected by maternal nutrition and dietary intake during pregnancy. Iron supplements starting at 4 months of age in breastfed infants, as recommended by the AAP, can offer a reasonable approach to reduce the risk of ID and IDA among Thai infants. Our results showed a significantly lower rate of ID and IDA among breastfed infants with iron supplementation, as well as those who were partially breast-fed and formula-fed, compared with infants who were breastfed and did not receive iron supplements. Our multivariate logistic regression analysis confirmed these findings and showed that iron supplementation among breastfed infants might reduce the risk of IDA by $90.4 \%$. In addition to iron supplements, evidence from a systematic review suggests that the introduction of solids at 4 months may have a beneficial effect on the rate of IDA in breastfed infants [29]. In our study, complementary foods were introduced to infants in each group between age 4 and 6 months at a different rate. However, complementary feeding did not seem to affect iron status, which showed no association with IDA in univariate analysis (Table 3). The absence of an association between complementary feeding and IDA may be owing to feeding practices in Thailand, where typical foods given to infants at this age are usually plant-based regarding the types of foods given to infants. Universal iron supplementation of breastfed infants at an early age is controversial. Iron homeostasis in infants younger than age 6 months is limited compared with older infants [31]. Iron absorption is not effectively downregulated in infants at an early age, who have adequate body iron; this raises concerns about iron toxicity or other side effects of iron supplementation among iron-sufficient infants. A systematic review and meta-analysis in 2013 found that daily iron supplementation impaired length gain and weight gain over the follow-up period, despite no differences in final weight or length [32]. Despite some concerns regarding infants' growth with daily iron supplementation, we found no significant 
differences in growth parameters among infants with different feeding patterns. However, our study included a relatively small sample size, and follow-up assessment was performed over a short period. Thus, we cannot support nor refute the above concerns. Adequately powered trials are needed to investigate the non-hematological benefits and risks of iron supplementation in this patient population. As an alternative to iron supplementation, measures to increase iron stores at birth among infants so as to provide them with adequate iron until late infancy, such as delayed cord clamping, have been reported [33].

This study has some limitations. Our study was performed at a single center with a limited sample size, so it was not sufficiently powered to be able to adequately assess the non-hematological effects of different feeding interventions. This study used retrospective data collected from electronic medical records; maternal baseline information during pregnancy and delivery as well as details regarding complementary foods given to infants were lacking. Information about feeding practices was based solely on parents' responses to questionnaires and interviews, which could have resulted in recall bias. Compliance with prescribed iron supplements was difficult to assess and could have resulted in misclassification bias. In our study, a simultaneous measurement of C-reactive protein to rule out infection was not performed at the time of laboratory serum ferritin measurement, as recommended by the AAP [14]. An elevated serum ferritin level in some infants in our study might indicate conditions such as inflammation, infection, malignancy, or liver disease rather than total body iron level. However, it is unlikely that infants had any of these conditions at well-child health maintenance visits, when laboratory testing was performed. Most participants in this study lived in Samut Prakan Province, which is located on the outskirts of Bangkok. Therefore, the data may not be representative of populations in other areas of Thailand. 


\section{Conclusions}

Anemia is not uncommon among Thai infants at 6 months of age. In our study,

365

366

367

368

369

370

371

372

373

374

375

376

377

378

379

380

381

382

383

384

385

386

387

significantly higher incidence of ID and IDA was found in Thai breastfed than formula-fed or partially breastfed infants. However, iron supplementation of breastfed infants with 1-2 $\mathrm{mg} / \mathrm{kg} /$ day starting at 4 months of age significantly decreased the incidence of ID and IDA.

8

\section{Abbreviations}

$\mathrm{Hb}$ - hemoglobin

HCT - hematocrit

ID - iron deficiency

IDA - iron deficiency anemia

$\mathrm{MCH}$ - mean corpuscular hemoglobin

MCHC - mean corpuscular hemoglobin concentration

MCV - mean corpuscular volume

RDW - red blood cell distribution width

\section{Acknowledgments}

We thank all the parents for bringing their infants to our hospital and allowing us to provide the best service possible for them. We especially thank the interns, pediatric hospitalists, and attending staff at CNMI for their hard work in performing patient follow-up in the well-baby clinic at CNMI. We would like to thank Assoc. Prof. Sasivimol Rattanasiri and Mrs.

Umaporn Udomsubpayakul for their assistance in the statistical analysis. We are grateful to Dr. Kulnipa Kittisakmontri for reviewing our manuscript and providing valuable suggestions. 


\section{Authors' contributions}

389

390

391

392

393

394

395

396

397

398

399

400

401

402

403

404

405

406

407

408

409

410

\section{Competing interests}

412 The authors declare no conflicts of interest.

\section{Availability of data and materials} corresponding author on reasonable request.

\section{Declarations}

\section{Ethics approval and consent to participate} patient informed consent was granted.

\section{Consent for publication}

Not applicable.

The datasets used and/or analysed during the current study are available from the

This study was approved by the Faculty of Medicine Ramathibodi Hospital, Mahidol University Ethics Committee (register No. MURA2021/44). The study was performed in accordance with the International Ethical Guidelines for Biomedical Research Involving Human Subjects and ethical principles of the Declaration of Helsinki. A waiver of individual 


\section{References}

414 1. Pasricha SR, Tye-Din J, Muckenthaler MU, Swinkels DW. Iron deficiency. Lancet. 2021;397(10270):233-248. doi:10.1016/S0140-6736(20)32594-0.

2. Kassebaum NJ, Jasrasaria R, Naghavi M, Wulf SK, Johns N, Lozano R, et al. A systematic analysis of global anemia burden from 1990 to 2010. Blood. 2014;123(5):615-624. doi:10.1182/blood-2013-06-508325.

3. Petry N, Olofin I, Hurrell RF, Boy E, Wirth JP, Moursi M, et al. The proportion of anemia associated with iron deficiency in low, medium, and high Human Development Index countries: a systematic analysis of national surveys. Nutrients. 2016;8(11):693. Published 2016 Nov 2. doi:10.3390/nu8110693.

4. Özdemir N. Iron deficiency anemia from diagnosis to treatment in children. Turk Pediatri Ars. 2015;50(1):11-19. Published 2015 March 1st. doi:10.5152/tpa.2015.2337.

5. Lozoff B, Brittenham GM, Wolf AW, McClish DK, Kuhnert PM, Jimenez E, et al. Iron deficiency anemia and iron therapy effects on infant developmental test performance [published correction appears in Pediatrics. 1988 May;81(5):683]. Pediatrics. 1987;79(6):981-995.

6. Lozoff B, Beard J, Connor J, Barbara F, Georgieff M, Schallert T. Long-lasting neural and behavioral effects of iron deficiency in infancy. Nutr Rev. 2006;64(5 Pt 2):S34-91.

7. WHO/UNICEF. Global strategy for infant and young child feeding. Geneva: World Health Organization; 2003.

8. Phuakpet K. Chapter 14: Screening for anemia. [in Thai] In: Noipayak P, Piyasil V, Ningsanon W, Prasopsri U. Guideline in child health supervision. Bangkok: Sappasan; 2014. p.152-60.

9. Bureau of Health Promotion, Department of Health, Ministry of Public Health, Thailand. Mother and Child Health Handbook 2555 (2012). 2012. 
10. Pizzaro F, Yip R, Dallman PR, Oliveres M, Hartrampf E, Walter T. Iron status with different infant feeding regimens: relevance to screening and prevention of iron deficiency. J Pediatr. 1991;118:687-92.

11. Calvo EB, Galindo AC, Aspres NB. Iron status in exclusively breastfed infants. Pediatrics. 1992;90:375-9.

12. Krishnaswamy S, Bhattarai D, Bharti B, Bhatia P, Das R, Bansal D. Iron deficiency and iron deficiency anemia in 3-5 months-old, breastfed healthy infants. Indian J Pediatr. 2017;84(7):505-508. doi:10.1007/s12098-017-2330-4.

13. Marques RF, Taddei JA, Lopez FA, Braga JA. Breastfeeding exclusively and iron deficiency anemia during the first 6 months of age. Rev Assoc Med Bras (1992). 2014;60(1):18-22. doi:10.1590/1806-9282.60.01.006.

14. Baker RD, Greer FR; Committee on Nutrition American Academy of Pediatrics. Diagnosis and prevention of iron deficiency and iron-deficiency anemia in infants and young children (0-3 years of age). Pediatrics. 2010;126(5):1040-50.

15. The Royal College of Pediatricians of Thailand \& Pediatric Society of Thailand. Guidelines for child health supervision from 0-18 years old. [in Thai]. Updated May 5, 2020. http://www.thaipediatrics.org/Media/media-20180329072750.pdf. Accessed 10 Sep 2021.

16. Chuansumrit A, Arnutti P, Apivanich S. Iron status of one-year-old infants in a well baby clinic. J Med Assoc Thai. 2002;85 Suppl 4:S1081-8.

17. Thaweekul P, Surapolchai P, Sinlapamongkolkul P. Infant feeding practices in relation to iron status and other possible nutritional deficiencies in Pathumthani, Thailand. Asia Pac J Clin Nutr. 2019;28(3):577-583. doi:10.6133/apjcn.201909_28(3).0017.

18. Tantracheewathorn S, Lohajaroensub S. Incidence and risk factors of iron deficiency anemia in term infants. J Med Assoc Thai. 2005;88(1):45-51. 
19. Suwannakeeree P, Jangmeonwai P. The prevalence and risk factors of iron deficiency anemia in Thai infants by complete blood count at 9-month-old. J Med Assoc Thai. 2020;103:891-6.

20. World Health Organization. Iron deficiency anemia. Assessment, prevention and control. A guide for programme managers. World Health Organization: Geneva, Switzerland, 2001; contract no.: WHO/NHD/01.3.

21. Rojroongwasinkul N, Kijboonchoo K, Wimonpeerapattana W, Purttiponthanee S, Yamborisut U, Boonpraderm A, et al. SEANUTS: the nutritional status and dietary intakes of 0.5-12-year-old Thai children. Br J Nutr. 2013;110 Suppl 3:S36-S44. doi:10.1017/S0007114513002110.

22. Nanta N, Natesirinilkul R, Kittisakmontri K, Chimnuan K, Manowong S, Suanta S, et al. Screening for iron deficiency anemia in infants in a thalassemia-endemic region. $\mathrm{J}$ Pediatr Hematol Oncol. 2021;43(1):e11-e14. doi:10.1097/MPH.0000000000001893.

23. Chaparro CM, Suchdev PS. Anemia epidemiology, pathophysiology, and etiology in low- and middle-income countries. Ann N Y Acad Sci. 2019;1450(1):15-31. doi:10.1111/nyas.14092.

24. Chen CM, Mu SC, Shih CK, Chen YL, Tsai LY, Kuo YT, et al. Iron status of infants in the first year of life in northern Taiwan. Nutrients. 2020;12(1):139. Published 2020 Jan 3. doi:10.3390/nu12010139.

25. Department of Health, Ministry of Public Health Thailand. Guidelines for the control and prevention of iron deficiency anemia. [in Thai]. https://www.nutrition.anamai.moph.go.th/images/file/filet001.pdf. Accessed 10 Sep 2021. 
26. Cai C, Granger M, Eck P, Friel J. Effect of daily iron supplementation in healthy exclusively breastfed infants: a systematic review with meta-analysis. Breastfeed Med. 2017;12(10):597-603. doi:10.1089/bfm.2017.0003.

27. Domellöf M, Cohen RJ, Dewey KG, Hernell O, Rivera LL, Lönnerdal B. Iron supplementation of breast-fed Honduran and Swedish infants from 4 to 9 months of age. J Pediatr. 2001;138(5):679-687. doi:10.1067/mpd.2001.112895.

28. Dumrongwongsiri O, Winichagoon P, Chongviriyaphan N, Suthutvoravut U, Grote V, Koletzko B. Zinc and iron adequacy and relative importance of zinc/iron storage and intakes among breastfed infants. Matern Child Nutr. 2021, e13268. doi.org/10.1111/mcn.13268.

29. Qasem W, Fenton T, Friel J. Age of introduction of first complementary feeding for infants: a systematic review. BMC Pediatr. 2015; September 2nd;15:107. doi: 10.1186/s12887-015-0409-5.

30. Kittisakmontri K, Fewtrell M, Roekworachai K, Phanpong C, Lanigan J. Complementary feeding: attitudes, knowledge and practices of urban families in northern Thailand. Nutr Diet. 2019;76(1):57-66. doi:10.1111/1747-0080.12474.

31. Lönnerdal B. Development of iron homeostasis in infants and young children. Am J Clin Nutr. 2017;106(Suppl 6):1575S-1580S. doi:10.3945/ajen.117.155820.

32. Pasricha SR, Hayes E, Kalumba K, Biggs BA. Effect of daily iron supplementation on health in children aged 4-23 months: a systematic review and meta-analysis of randomised controlled trials [published correction appears in Lancet Glob Health. 2014;2(3):e144]. Lancet Glob Health. 2013;1:e77-86.

33. Kc A, Rana N, Målqvist M, Ranneberg LJ, Subedi K, Andersson O. Effects of delayed umbilical cord clamping vs early clamping on anemia in infants at 8 and 12 months: a 
randomized clinical trial. JAMA Pediatr. 2017;171(3):264-270.

511 doi:10.1001/jamapediatrics.2016.3971.

512 


\section{Excluded} - 5 specimens tested more than 1 time in the same patient at 5-7 months of age

3

.

4

25

26

227
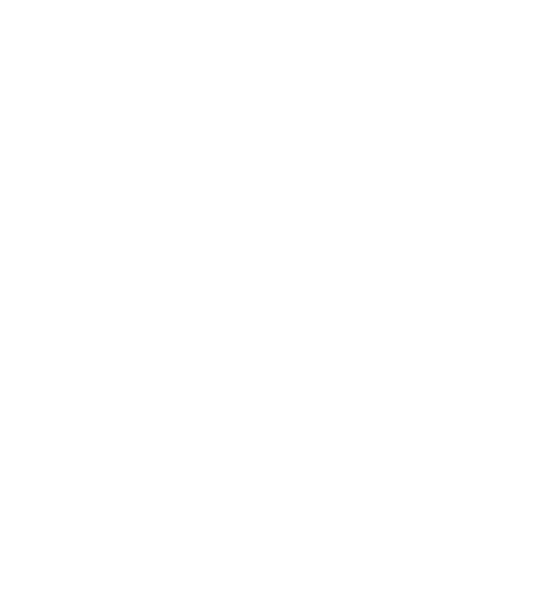

\section{Serum ferritin measurement during}

1 January 2019 to 31 Dec 2020 $\mathrm{N}=2433$ (specimens)

\section{Excluded}

- 1992 specimens of adults and children outside of age ranges

\section{Excluded}

-15 infants gestational age $<35$ weeks

- 13 infants gestational age $\geq 35$ weeks who received iron supplements before 4month visit

- 5 infants with incomplete medical records complete blood count) $\mathrm{N}=403$ (patients)

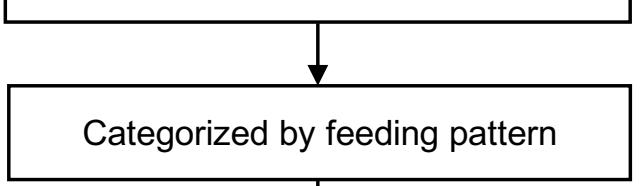

.

\begin{tabular}{|c|c|c|c|}
\hline $\begin{array}{c}\text { Breastfed } \\
(\mathrm{BF})\end{array}$ & $\begin{array}{c}\text { Breastfed with } \\
\text { iron } \\
\text { supplements } \\
(\mathrm{BI})\end{array}$ & $\begin{array}{c}\text { Mixed-fed } \\
\text { (breast milk and } \\
\text { formula) with or } \\
\text { without iron } \\
\text { supplements } \\
\text { (MF) } \\
\mathrm{N}=109\end{array}$ & $\begin{array}{c}\text { Formula-fed } \\
\text { (FF) }\end{array}$ \\
$\mathrm{N}=78$ & $\mathrm{~N}=111$ \\
\hline
\end{tabular}


Table 1. Baseline characteristics of infants in the study

\begin{tabular}{|c|c|c|c|c|c|}
\hline Characteristics & $\begin{array}{c}\text { Breastfed } \\
\text { (BF) } \\
(\mathrm{N}=105)\end{array}$ & $\begin{array}{c}\text { Breastfed with } \\
\text { iron } \\
\text { supplements } \\
(\mathrm{BI}) \\
(\mathrm{N}=78) \\
\end{array}$ & $\begin{array}{c}\text { Mixed-fed with } \\
\text { or without iron } \\
\text { supplements } \\
(\mathrm{MF}) \\
(\mathrm{N}=109) \\
\end{array}$ & $\begin{array}{c}\text { Formula-fed } \\
\text { (FF) } \\
(\mathrm{N}=111) \\
\end{array}$ & p-value \\
\hline Male sex & $57(54.3)$ & $37(47.4)$ & $59(54.1)$ & $52(46.9)$ & 0.564 \\
\hline Inborn & $43(41.0)$ & $33(42.3)$ & $49(45.0)$ & $42(37.8)$ & 0.757 \\
\hline Gestational age (weeks) & $38 \pm 1$ & $38 \pm 1$ & $38 \pm 1$ & $38 \pm 1$ & 0.521 \\
\hline Small for gestational age & $13(12.4)$ & $18(23.1)$ & $12(11.0)$ & $15(13.5)$ & 0.127 \\
\hline Large for gestational age & $0(0)$ & $3(3.9)$ & $0(0)$ & $5(4.5)$ & 0.008 \\
\hline \multicolumn{6}{|l|}{ At birth } \\
\hline Weight $(\mathrm{g})$ & $3103 \pm 391$ & $3105 \pm 461$ & $3120 \pm 397$ & $3195 \pm 449$ & 0.251 \\
\hline Length $(\mathrm{cm})$ & $49.5 \pm 2.2$ & $49.2 \pm 2.1$ & $49.6 \pm 2.2$ & $50.1 \pm 2.2$ & 0.971 \\
\hline Head circumference $(\mathrm{cm})$ & $33.6 \pm 1.4$ & $33.7 \pm 1.4$ & $33.7 \pm 1.2$ & $34.0 \pm 1.4$ & 0.411 \\
\hline \multicolumn{6}{|l|}{ At 4-month visit } \\
\hline Weight $(\mathrm{g})$ & $6606 \pm 813$ & $6569 \pm 905$ & $6598 \pm 895$ & $6727 \pm 842$ & 0.699 \\
\hline Length $(\mathrm{cm})$ & $62.8 \pm 2.2$ & $62.7 \pm 2.5$ & $63.0 \pm 2.3$ & $63.4 \pm 2.2$ & 0.681 \\
\hline Head circumference $(\mathrm{cm})$ & $41.0 \pm 1.2$ & $40.8 \pm 1.4$ & $41.0 \pm 1.4$ & $40.9 \pm 1.3$ & 0.675 \\
\hline \multicolumn{6}{|l|}{ At 6-month visit } \\
\hline Weight $(\mathrm{g})$ & $7468 \pm 853$ & $7475 \pm 1065$ & $7603 \pm 1007$ & $7730 \pm 924$ & 0.158 \\
\hline Length $(\mathrm{cm})$ & $66.1 \pm 2.3$ & $66.2 \pm 2.6$ & $66.8 \pm 2.3$ & $67.3 \pm 2.4$ & 0.523 \\
\hline Head circumference $(\mathrm{cm})$ & $42.7 \pm 1.3$ & $42.5 \pm 1.4$ & $42.7 \pm 1.5$ & $42.6 \pm 1.2$ & 0.327 \\
\hline $\begin{array}{l}\text { Complementary feeding } \\
\text { before } 6 \text { months }\end{array}$ & $36(34.3)$ & $41(52.6)$ & $87(79.8)$ & $95(85.6)$ & $<0.001$ \\
\hline Iron supplementation & $0(0)$ & $78(100.0)$ & $40(36.7)$ & $0(0)$ & $<0.001$ \\
\hline
\end{tabular}

538 Values in the table are $\mathrm{n}(\%)$ or mean \pm standard deviation.

539

540

541

542

543

544

545

546

547

548

549

550

551

552 
Table 2. Laboratory characteristics of infants categorized by feeding practices

\begin{tabular}{|c|c|c|c|c|c|}
\hline Characteristics & $\begin{array}{c}\text { Breastfed } \\
\text { (BF) }\end{array}$ & $\begin{array}{c}\text { Breastfed with } \\
\text { iron } \\
\text { supplements } \\
(\mathrm{BI}) \\
(\mathrm{N}=\mathbf{7 8})\end{array}$ & $\begin{array}{c}\text { Mixed-fed with } \\
\text { or without iron } \\
\text { supplements } \\
(\mathrm{MF}) \\
(\mathrm{N}=109)\end{array}$ & $\begin{array}{c}\text { Formula-fed } \\
\text { (FF) }\end{array}$ & p-value \\
\hline $\mathrm{Hb}(\mathrm{g} / \mathrm{dL})$ & $11.1 \pm 1.0^{\mathrm{a}}$ & $11.7 \pm 1.0$ & $11.8 \pm 0.9$ & $11.8 \pm 0.9$ & $<0.001$ \\
\hline $\operatorname{HCT}(\%)$ & $34.4 \pm 2.9^{\mathrm{b}}$ & $36.2 \pm 3.0$ & $36.2 \pm 2.6$ & $35.3 \pm 2.6$ & $<0.001$ \\
\hline $\mathrm{MCV}(\mathrm{fL})$ & $69.8 \pm 6.9^{\mathrm{c}}$ & $71.8 \pm 7.1$ & $73.7 \pm 6.5$ & $74.3 \pm 6.0$ & $<0.001$ \\
\hline $\mathrm{MCH}(\mathrm{pg})$ & $22.6 \pm 2.5^{\mathrm{c}}$ & $23.4 \pm 2.7^{\mathrm{e}}$ & $24.1 \pm 2.4$ & $24.8 \pm 2.2$ & $<0.001$ \\
\hline $\operatorname{MCHC}(\mathrm{g} / \mathrm{dL})$ & $32.3 \pm 1.1^{\mathrm{e}}$ & $32.5 \pm 1.0^{\mathrm{e}}$ & $32.7 \pm 1.1^{\mathrm{e}}$ & $33.3 \pm 1.0$ & $<0.001$ \\
\hline RDW (\%) & $14.6 \pm 2.3^{\mathrm{e}}$ & $14.5 \pm 2.2^{\mathrm{e}}$ & $14.0 \pm 2.1$ & $13.4 \pm 1.9$ & $<0.001$ \\
\hline Serum ferritin $(\mathrm{ng} / \mathrm{mL})$ & $37.5 \pm 43.4^{\mathrm{a}}$ & $88.7 \pm 84.1$ & $74.8 \pm 53.6$ & $80.2 \pm 47.6$ & $<0.001$ \\
\hline $\begin{array}{l}\text { ID (Serum ferritin }<12 \\
\mathrm{ng} / \mathrm{mL} \text { ) }\end{array}$ & $30(28.6)^{a \dagger}$ & $3(3.8)$ & $4(3.7)$ & $1(0.9)$ & $<0.001$ \\
\hline Anemia $(\mathrm{Hb}<11 \mathrm{~g} / \mathrm{dL})$ & $40(38.1)^{\mathrm{c}^{\dagger}}$ & $17(21.8)$ & $21(19.3)$ & $18(16.2)$ & $<0.001$ \\
\hline IDA & $18(17.1)^{\mathrm{a} \dagger}$ & $2(2.6)$ & $1(0.9)$ & $1(0.9)$ & $<0.001$ \\
\hline ID without anemia & $12(11.4)^{\mathrm{d} \dagger}$ & $1(1.3)$ & $3(2.8)$ & $0(0)$ & $<0.001$ \\
\hline Anemia without ID & $22(21.0)$ & $15(19.2)$ & $20(18.3)$ & $17(15.3)$ & 0.754 \\
\hline
\end{tabular}

$555 \quad$ Values in the table are $\mathrm{n}(\%)$ or mean \pm standard deviation.

556 a - significant difference with BI, MF, FF; b - significant difference with BI, MF; c - significant

557 difference with MF, FF; d - significant difference with BI, FF; e - significant difference with FF.

$558 \dagger$ significant difference with $\mathrm{p}$-value $<0.008$.

$559 \mathrm{Hb}$ - hemoglobin, HCT - hematocrit, ID - iron deficiency, IDA - iron deficiency anemia, MCV - mean

560 corpuscular volume, $\mathrm{MCH}$ - mean corpuscular hemoglobin, $\mathrm{MCHC}$ - mean corpuscular hemoglobin

561 concentration, RDW - red blood cell distribution width.

562

563

564

565

566

567

568

569

570

571

572

573

574 
Table 3. Logistic regression analyses of factors associated with iron deficiency anemia in

\section{6-month-old infants}

\begin{tabular}{|l|c|c|c|c|c|c|}
\hline \multicolumn{1}{|c|}{ Variables } & $\begin{array}{c}\text { Crude } \\
\text { OR }\end{array}$ & $\mathbf{9 5 \%}$ CI & p-value & $\begin{array}{c}\text { Adjusted } \\
\text { OR }\end{array}$ & 95\% CI & p-value \\
\hline Gestational age & 0.586 & $0.392-0.875$ & 0.009 & 0.819 & $0.464-1.447$ & 0.819 \\
\hline Birth weight & 0.998 & $0.997-0.999$ & 0.003 & 0.997 & $0.995-0.999$ & 0.002 \\
\hline Male sex & 2.709 & $1.038-7.072$ & 0.042 & 2.585 & $0.818-8.168$ & 0.106 \\
\hline Weight gain 0-6 months & 1.001 & $1.000-1.001$ & 0.011 & 1.001 & $1.000-1.001$ & 0.010 \\
\hline Small for gestational age & 1.346 & $0.439-4.128$ & 0.604 & & & \\
\hline $\begin{array}{l}\text { Complementary feeding } \\
\text { before 6 months }\end{array}$ & 0.651 & $0.274-1.546$ & 0.331 & & & \\
\hline Feeding practices & \multicolumn{7}{|l|}{} & $1($ ref) & & \\
\hline BF & 1 (ref) & \multicolumn{7}{|l|}{0.007} & \\
\hline BI & 0.127 & $0.029-0.566$ & 0.007 & 0.096 & $0.020-0.466$ & 0.004 \\
\hline MF & 0.045 & $0.006-0.342$ & 0.003 & 0.025 & $0.004-0.267$ & $<0.001$ \\
\hline FF & 0.044 & $0.006-0.336$ & 0.003 & 0.031 & $0.003-0.218$ & 0.002 \\
\hline
\end{tabular}

